\title{
Pilot's Attention Distributions between Chasing a Moving Target and
} a Stationary Target

Wen-Chin Li ${ }^{1 *}$, Chung-San $\mathrm{Yu}^{2}$, Graham Braithwaite ${ }^{1}$ \& Matthew Greaves ${ }^{1}$

${ }^{1}$ Safety and Accident Investigation Centre, Cranfield University Cranfield, Beds MK43 OAL, United Kingdom

${ }^{2}$ Department of Industrial Engineering and Engineering Management, National Tsing Hua University

101 Sec. 2, Kuang-Fu Rd. Engineering Building 1, RM 711, Hsinchu City 30013, Taiwan, Republic of China

Tel: $\quad+44$ (1234) 758527 (Wen-Chin Li, PhD)

+886 (3) 5742929 (Chung-San Yu, PhD)

+44 (1234) 754252 (Graham Braithwaite, PhD)

+44 (1234) 754243 (Matthew Greaves, PhD)

Fax: $\quad+44(1234) 758527$

E-mail: wenchin.li@cranfield.ac.uk (W-C. Li)

E-mail: mountainsyudyahoo.com (C-S. Yu)

E-mail: g.r.braithwaitedcranfield.ac.uk (G. Braithwaite)

E-mail: m.j.greaves@cranfield.ac.uk (M. Greaves)

Running Head: Pilots' Attention Distributions to Chase Different Targets

Manuscript metrics

Words count for Abstract: 250

Words count for Narrative: 3350

Number of References: 24

Number of Tables: 3

Number of Figures: 4 
1 ABSTRACT

2 Introduction: Attention plays a central role in cognitive processing;

3 ineffective attention may induce accidents in flight operations. The

4 objective of current research was to examine military pilots' 5 attention distributions between chasing a moving target and a

6 stationary target. Method: Thirty-seven mission-ready F-16 pilots

7 participated in the current research. Subjects' eye movements were

8 collected by a portable head-mounted eye-tracker during tactical

9 training in a flight simulator. The scenarios of chasing a moving

10 target (air-to-air) and a stationary target (air-to-surface) consist

11 of three operational phases; searching, aiming and lock-on to the

12 targets. Results: The findings demonstrated significant differences

13 in pilots' percentage of fixation during searching phase between 14 air-to-air $(\mathrm{M}=37.57, \mathrm{SD}=5.72)$ and air-to-surface $(\mathrm{M}=33.54, \mathrm{SD}=4.68)$.

15 Fixation duration can indicate pilots' sustained attention to the

16 trajectory of a dynamic target during dog-fight manoeuvers. Aiming

17 for the stationary target with larger pupil size (M=27105 pixel²,

$18 \mathrm{SD}=6565 \mathrm{pixel}^{2}$ ) reflects higher cognitive loading than aiming to the

19 dynamic target ( $M=23864 \mathrm{pixel}^{2}, \mathrm{SD}=8762 \mathrm{pixel}^{2}$ ). Discussion: Pilots'

20 visual behavior is not only closely related to attention distribution,

21 but also significantly associated with task characteristics. Military

22 pilots demonstrated various visual scan patterns for searching and

23 aiming to different types of targets based on the research settings

24 of flight simulator. The findings would facilitate system designers'

25 understandings of military pilots' cognitive processes during

26 tactical operations. It will assist human-centered interface design

27 to improve pilots' situational awareness. The application of an

28 eye-tracking device integrated with a flight simulator is a feasible

29 and cost-effective intervention to improve efficiency and safety of

30 tactical training.

31

Keywords: attentional processes; eye movements; mental workload; 
1

\section{INTRODUCTION}

Pilots have to process information based on interior cockpit indicators and the exterior environmental stimuli by visual search during flight operations. Compared with commercial flight, exterior stimuli for military pilots also include either the moving target of a foe or a stationary surface target. Lavine, Sibert, Gokturk, and Dickens (12) suggest that visual attention is a precursor to initiate the cognitive process and information acquired from pilot's visual scan is closely associated with a pilot's attention allocation. Ineffective attention distribution may induce accidents (e.g., Asiana Airlines Flight 214 which crashed on final approach), as pilots' lack of situation awareness to the airspeed indicator was a critical human factors issue in the accident (17). Attention plays a central role in cognitive processing. How and where pilots distribute attention is critical to the quality of situational awareness (SA) and links to the features of individual's expectations (7). Therefore, eye movements may serve as a window to illustrate pilots' attention distribution and mental state during flight operations (13). The pattern of pilots' eye movement is one of the methods for assessing pilots' cognitive processes, based on real-time physiological measures (1). Therefore, pilots' visual behaviors are indicators to reveal attentional distributions during flight operations (9, 21). Fixation is defined typically as the eye movement pausing over informative regions of interest. Human beings usually retain fixations on the objects to acquire the most essential information to support the task in hand (21). The patterns of fixations on the indicators or the areas of interest (AOIs) can reveal a pilot's visual 
1 trajectory of attention (23). Moreover, the percentage of fixations 2 on the relevant AOIs is deemed as the predictor of the overall SA performance (15). In addition, the length of fixation duration is the

4 total time fixating on an AOI, which can reflect the level of

5 importance or difficulty in extracting information (2). Fixation

6 duration might reveal how long pilots sustain attention whilst

7 scanning the visual fields in order to complete the mission. On the

8 other side, fixation duration might be an index of cognitive capture

9 or over-concentration on a specific indicator, which will slow down

10 attention shifts to the tactical situation (7).

11 Pupil dilation is known to quickly respond to changes in the

12 illumination in the visual field and to a human being's perceived

13 workload while performing a visual task. Under controlled

14 illumination, the pupil size is an effective and reliable indicator

15 of mental workload. The increasing in pupil size is correlated with

16 the increasing in mental workload (6). Attention is critical to pilots

17 filtering the stimuli to the perceptual system. However, workload

18 usually has negative impacts to the effectiveness of visual attention

19 (14). The increasing pupil size is a physical feature of cognitive

20 load (19), as it can be an important indicator of a pilot's cognitive

21 process and visual attention (23).

22 Saccadic eye movements are controlled by top-down visual processes, 23 which are coordinated closely with perceptual attention (24). It

24 indicates that saccadic paths are intentional and meaningful based

25 on the requirements of the task in hand and the trajectory prediction

26 in the near future (11). Therefore, the path of saccades is associated

27 with selective attention and accurate judgments for perceptual 
1 targets $(4,16)$. Saccade duration is the total time taken to make a

2 saccade, which is recognized as one of indexes to assess operator's

3 workload; e.g., increase in workload has been found to decrease

4 saccade duration (20). Saccade velocity is how fast the eyes move

5 between fixations, which are associated with rapid deployment of

6 attention. Thus saccades might be an effective indicator of attention

7 distribution.

8 The information provided in the cockpit is mostly acquired by

9 pilots' visual scans among cockpit interfaces, and previous research

10 has shown that $75 \%$ of pilot errors result from poor perceptual encoding

$11(3,8)$. It highlights the importance of the interactions between

12 pilots' visual scan and the characteristics of cockpit interface

13 design. It is obviously that attention is a critical precursor to

14 in-flight SA performance and decision-making (18). Eye tracking has

15 been gaining in popularity over the past decade as a window into

16 participants' visual and cognitive processes. Therefore, analysis

17 metrics of current research include five parameters of visual behavior:

18 the percentage of fixations, fixation duration, pupil size, saccade

19 duration, and saccade velocity among three operational phases

20 composed with searching for visual contact with a target, aiming at

21 a target, and lock-on for pick-off (press the trigger to launch weapon)

22 between air-to-air for a moving target and air-to-surface for a

23 stationary target. Based on the above literature review, there are

24 four fundamental hypotheses will be investigated as followings:

25 (1) there is no significant difference in pilots' fixation duration

26 between chasing a moving target and a stationary target; (2) there

27 is no significant differences in pilots' fixation duration among three 
1 operational stages; (3) there is no significant difference on pilots'

2 pupil dilation between chasing a moving target and a stationary

3 target; (4) there is no significant differences on pilots' saccade

4 velocity among three operational stages.

5

\section{METHODS}

7 Aims

8 The research aims were (1) to investigate pilots' visual

9 characteristics between pursuing a moving and a stationary target;

10 (2) to explore pilots' eye movement patterns and attention

11 distributions on three operational stages, searching, aiming and

12 lock-on a target; (3) to evaluate pilots' pupil dilation and cognitive

13 process on three operational stages between the pursuit of a moving

14 and a stationary target; and (4) to apply the findings to benefit

15 military pilot training and cockpit interface design.

16

17 Subjects

18 A total of thirty-seven qualified mission-ready F-16 pilots

19 participated in this research. The subjects' flying experience varied

20 between 372 and 3,200 hours (M=1280, SD=769). The ages ranged between

2126 and 45 years old $(M=33, S D=5)$. All of the subjects were male

22 volunteers and informed that they had the right to cease the

23 experiments and withdraw information they provided without any reason.

24 Subjects signed an informed consent form and reported normal levels

25 of visual function. The treatment of all subjects complied with the

26 ethical standards required by the Research Ethics Regulations of

27 Cranfield University. 
2 Equipment

3 Flight Simulator: The flight simulator used in the experiment is

4 a formal F-16 trainer. It is a high-fidelity and fixed-base type flight

5 simulator. It consists of identical cockpit displays to those in the

6 actual aircraft to supports pilots' routine flight training and combat

7 planning. It is integrated with high-definition databases, image

8 generation systems and physics-based processing technology which

9 enable pilots to detect, judge the orientation of, recognize and

10 identify targets as they would in the real world of tactical

11 operations. The instructor can install scenarios and observe the

12 trainee pilot's performance via a console with three monitors.

13 Eye Tracking Device: Pilots' eye movement data were collected by

14 a mobile head-mounted eye-tracker which is designed by Applied Science

15 Laboratory (ASL Series 4000). It is portable and light (76 g) so

16 participants can move their head without any limitations. The sampling

17 frequency of this type of eye-tracker is $30 \mathrm{~Hz}$. Video recordings of

18 eye movements and the related data were collected and stored using

19 a Digital Video Cassette Recorder (DVCR) and then transferred to a

20 computer for further analysis. The definition of an eye fixation in

21 the present study was as three gaze points occurred within an area

22 of 10 by 10 pixels with a dwell time more than $200 \mathrm{msec}$ (21).

24 Scenarios

25 Air-to-Air Task for Pursuing a Moving Target: The scenario-1 is 26 an air-to-air (A-A) manoeuver to pursue a dynamic target. The altitude 27 of the interceptor (participant) at the patrol area was 20,000 feet 
1 with a cruise speed of 300 knots indicated airspeed (KIAS). The heading

2 was $050^{\circ}$ under the weather conditions of 7-mile visibility and scattered clouds. A foe unexpectedly appears at the same altitude as the target moving from left to right with heading of $090^{\circ}$ and air speed of 300 KIAS. The participants have to search the airspace for the target, and intercept the target immediately by tactical manoeuvers . At the same time, the target would change its heading, altitude and speed in order to escape from the interceptor's pursuit (figure la).

9

[Figure 1 here]

11

12

Air-to-Surface Task for Aiming at a Stationary Target: The scenario-2 is an air-to-surface ( $\mathrm{A}-\mathrm{S})$ manoeuver to pursue a stationary

14 target. Participants were dispatched unexpectedly to attack one stationary target, where they not only needed to execute tasks

16 precisely by operating the aircraft, but also to follow the navigation 17 system, entering appropriate codes by using various cockpit

18 interfaces. Participants had to intercept the proper route and turn 19 toward the target at an altitude of 500 feet with a speed of 500 KIAS 20 simultaneously, then performed a steep pop-up manoeuver to increase altitude abruptly for appropriate target reconnaissance, followed by a dive and roll-in toward the surface target to avoid hostile radar lock-on. When approaching the target, participants have to roll-out, level the aircraft, aim at the target, lock-on and pick-off the target (figure 1b).

Research Design 
Procedures: All participants undertook the following procedures;

(1) complete the demographical data including rank, job title, age, education level, qualifications, type hours and total flight hours

(2) a short briefing explaining the purpose of the study and the introduction of the air-to-air and air-to-surface scenarios without mentioning any potential aircraft equipment failure (20 minutes); (3) participants were seated in the F-16 simulator and then the eye-tracker was put on for calibration using three points distributed over the cockpit display panels and outer screen (15-25 minutes) ; (4) perform the air-to-air task for aiming at a dynamic target (5 minutes); (5) perform air-to-surface task for aiming at a stationary target on the ground (5 minutes); simultaneously the instructor pilot in the simulator console evaluated participants' performance. It took around 60 minutes for each participant to complete the experiments. Analysis of Eye Movements Data: The eye movement data of both air-to-air and air-to-surface tasks in this study were analyzed by three phases of visual behavior during tactical operations: searching

19 for the target with eye contact (Searching), pursuing the target for 20 aiming (Aiming), and lock-on to the target for pick-off (Lock-on). The length of time for analyzing each operational phase was 6 seconds (18 seconds in total for three phases). It was grounded by the consensus of experienced instructor pilots based on the most critical decisive time to process tactical information during performing both air-to-air and air-to-surface tasks. The variables of eye movement data were analyzed by percentage of fixation, fixation duration, pupil size, saccade duration and saccade velocity. 
1

\section{RESULTS}

3

4

5

6

7

8

9

10

15

16

17
The demographical information of participants' age, rank, qualification and total flight hours are shown as table I. As percentage of fixation is proportional data, it is necessary to perform an arcsine transformation in advance to enable further statistical analysis (5). Based on the research design of current study, a paired T-test and ANOVA were applied to analyze the differences of eye movement data between air-to-air and air-to-surface during three operational phases of searching, aiming and lock-on (dependent variables). The analysis for this study is a within subjects test, as all participants were performing both tactical tasks of aiming at a dynamic target (air-to-air) and a stationary target (air-to-surface).

[Table I here]

There were five dependent variables related to pilots' eye movement characteristics between air-to-air and air-to-surface tasks among three operational phases, which are fixations/ percentage of fixation, fixation duration, pupil size, saccade duration, and saccade velocity. The results demonstrated that there were significant differences in pilots' fixations ( $t=-2.52, p<.05, d=-.624)$ and fixation duration $(t=3.26, p<.005, d=.748)$ between air-to-air and air-to-surface task. Therefore, the null hypothesis 'there is no significant differences on pilots' fixation duration between chasing a moving target and a stationary target' was rejected. Also, there were significant 
1 differences in pilots' saccade duration between the two tasks, $t=-2.30$,

$2 p<.05, d=-.372$. However, there were no significant differences in

3 pilots' pupil size ( $t=-1.92, p>.05, d=-.252)$ and saccade velocity

$4(t=-1.31, p>.05, d=-.214)$ between two tasks (table II).

5

$6 \quad[$ Table II here $]$

7

8 Significant differences among three operational phases were

9 observed in terms of percentage of fixation during air-to-air, F (2,

$1036)=5.75, \mathrm{p}<.01, \mathrm{\eta} 2 \mathrm{p}=.138$, and air-to-surface, $\mathrm{F}(2,36)=6.29, \mathrm{p}<.01$,

$11 \eta 2 p=.149$. Further comparisons by post-hoc Bonferroni adjusted tests showed that during air-to-air task, searching (37.57) has a higher percentage of fixations than aiming (35.11), and lock-on (32.94); the

14 highest percentage of fixations was occurred at aiming phase during air-to-surface. There were significant differences in pilots'

16 fixation duration among three operational phases at air-to-air, F (2, $1736)=5.39, p<.01, \eta 2 \rho=.130$, and also at air-to-surface, $F(2,36)$

$18=18.48, p<.001, \eta 2 \rho=.339$. Further comparisons by post-hoc Bonferroni

19 adjusted tests showed that lock-on (938 msec) has significantly longer

20 fixation duration than aiming (702 $\mathrm{msec}$ ) and searching (612 $\mathrm{msec}$ )

21 during air-to-air task; the patterns showed at air-to-surface was same

22 as air-to-air, lock-on the longest fixation duration (580 msec), then

23 aiming (462 $\mathrm{msec}$ ) and searching (332 $\mathrm{msec})$ (table III). Therefore,

24 the null hypothesis 'there is no significant differences on pilots'

25 fixation duration among three operational stages' was rejected. 
1
There were significant differences in pilots' pupil dilation among three phases during air-to-air, $F(2,36)=7.57, p<.01, \eta 2 p=.174$, and air-to-surface, $F(2,36)=38.82, p<.001, \eta 2 p=.519$. Further comparisons by post-hoc Bonferroni adjusted tests showed that pilots' largest pupil size at air-to-air was in the phase of lock-on (26147 pixel ${ }^{2}$ ); the largest one at air-to-surface was occurred in aiming (27105 pixel ${ }^{2}$ ). Therefore, the null hypothesis 'there is no significant differences on pilots' pupil dilation between chasing a moving target and a stationary target' was rejected. There were significant differences in pilots' saccade velocity among the three phases during air-to-surface tasks, F $(2,36)=7.87$, $\mathrm{p}<.01, \mathrm{\eta} 2 \mathrm{p}=.179$. Further comparisons by post-hoc Bonferroni adjusted tests showed that pilots' saccade velocity during air-to-surface task at the phase of lock-on (1148 pixels/sec) was significantly longer than at aiming (1045 pixels/sec) and at searching (829 pixels/sec). However, there were no significant differences in pilots' saccade velocity among three phases during air-to-air task, $F(2,36)=.68$, p>.05, $\eta 2 p=.019$ (table III). Therefore, the null hypothesis 'there is no significant differences on pilots' saccade velocity among three operational stages' was partially rejected.

\section{DISCUSSION}

The characteristics of the air-to-air task in current study are engaging a dynamic target by visual searching to aim and lock-on the moving target. On the other hand of air-to-surface, pilots have to perform a steep pop-up manoeuver to search for the target, followed 
1 by a rapid dive and roll-in to aim and lock-on the stationary target.

2 The results showed the significant differences in pilots' fixations and fixation duration between the pursuit of a moving and a stationary

4 target (table II). Pilots did demonstrate different patterns of

5 fixations and fixation duration between chasing a moving target and

6 stationary target. Furthermore, pilot's in-flight cognitive process

7 is extremely dynamic, which needs to be explored by the contexts of

8 operational environment.

9 Two different tactical tasks in current study are composed with

10 three operational phases; each phase has specific tactical

11 requirements and threats. Table III shows pilots distributed the

12 highest percentage of fixations on aiming at the surface target (37.62

13 arcsine values). It reflects the tactical standard operating

14 procedures that pilots have to precisely aim at the surface target

15 within the time frame (between 3-5 seconds), otherwise the mission

16 would be aborted. On the other side, searching a moving target at

17 air-to-air task represents the highest percentage of fixations (37.57

18 arcsine values), which demonstrates that the uncertain trajectory of

19 a moving target might increase pilots' cognitive load in searching

20 for the unknown airborne target.

21 Pilots' fixation duration during the air-to-air task was

22 significantly longer than the air-to-surface task across all phases

23 (table III). It might indicate that pilots have to sustain substantial

24 attention to avoid missing the trajectory of a dynamic target during

25 the high kinetic manoeuvers. Especially the interval (236 msec) from

26 aiming to lock-on, pilots' fixation duration increased 2.6 times

27 compared to the interval from searching to aiming ( $90 \mathrm{msec}$ ). It reveals 
1 that pilots have to keep tracking and precisely project the target's

2 probable trajectory movement in the vast airspace while aiming and

3 locking-on a dynamic target.

4

[Figure 2 here]

6

Figure 2 indicates that pilots' pupil size in the phase of lock-on $\left(26,147 \mathrm{pixel}^{2}\right)$ is the greatest at the pursuit of a moving target. Also, the tendency of increasing pupil dilation along task performance might reveal pilots' increasing cognitive load from searching to lock-on. However, the pupil size at the pursuit of a stationary target is averagely greater than at the moving target. Figure 2 also shows the greatest pupil size was occurred at the aiming phase. The results did reveal there are significant differences on pilots' pupil dilation among three operational stages. Also, the increasing in pupil dilation from searching to aiming during the air-to-surface $\left(3,108\right.$ pixel $\left.^{2}\right)$ is significantly greater than air-to-air (1,904 pixel $\left.{ }^{2}\right)$. It shows that pilots might have tremendous cognitive workload during the air-to-surface task compared with air-to-air. The findings are constructive to comprehend pilots' cognitive processes regarding the aspect of workload objectively while chasing a stationary target with potential accident of control flight into terrain (CFIT) (10).

[Figure 3 here]

The significant difference in pilots' saccade duration was observed between the air-to-air and air-to-surface tasks (table II). 
1 Figure 3 reveals that pilots significantly decreased time to make a 2 saccade while searching a dynamic target (239 $\mathrm{msec})$ than searching 3 for a stationary target (457 $\mathrm{msec})$. It illustrated that pilots shifted

4 attention with shorter time to search for an almost unknown and moving 5 target than for a stationary target with awareness of approximate 6 location. As a result, the level of knowledge of the target influences 7 pilot's saccade duration. In addition, the saccadic duration is 8 accompanied by a shift of attention to the selected target (11). 9 Searching for the stationary surface target seems to reflect higher 10 cognitive load than searching for the dynamic target (20). Pilots 11 operating fighter aircraft towards a surface target must fly so 12 precisely in order to avoid the accident of CFIT. Simultaneously, they 13 also have to be aware of hostile threats while assessing appropriate 14 timing for lock-on and pick-off. It was found that the decreasing rate at saccade duration from searching to aiming during the air-to-surface

16 task is $55.36 \%$ (figure 3 ).

17

18 [Figure 4 here]

19

There was no significant difference between two tasks although table II reveals average saccade velocity at the pursuit of a stationary target (1007 pixels/ sec) is faster than the pursuit of a moving target (948 pixels/ sec). However, there were significant differences among three phases during the air-to-surface task (table III). Figure 4 reveals the fastest saccade velocity was occurred at the lock-on phase (1148 pixels/sec). In contrast, the slowest saccade 
1 of collecting relevant navigation and target information for further

2 operations. Processing massive amounts of information inducing high cognitive load might be the reason to make the searching phase

4 demonstrating the slowest saccade velocity and the longest saccade

5 duration. In addition, the fastest saccade velocity reveals the

6 lock-on phase requiring quick attention shifts to enhance situational

7 awareness as flying at extreme low altitude for air-to-surface task.

8 The findings of saccade duration and saccade velocity reveal pilots'

9 top-down visual scan patterns in tactical operations based on pilots'

10 expectations (projection of the course of action) associated with

11 specific objectives which are matched with the previous research (4, 12 22).

13

14 CONCLUSION

15 Current research found that pilots would apply different

16 approaches of visual scan patterns for searching and lock-on to

17 different types of targets. Eye tracking devices can aid in capturing

18 a pilot's attention allocation where traditional flight simulators

19 training were lacking. Additionally, the analysis of eye movement

20 parameters in real-time tactical manoeuvers could provide system designers with a better understanding of the tendency of pilots' cognitive process to optimize interface design and alleviate pilots' workload. The findings of current research also could facilitate the development of tactical training syllabi for air-to-air and air-to-surface tasks to improve pilots' attention distribution and situational awareness. However, the present findings were based on experiments conducted in a ground-based flight simulator. In order 
1 to reflect military pilots' in-flight cognitive process, next step 2 is to develop a cockpit eye tracker to further study pilots' eye 4 operations.

5 6 


\section{REFERENCES}

2 1. Di Stasi LL, Antolí A, Cañas JJ. Main sequence: An index for 3

4 detecting mental workload variation in complex tasks. Appl Ergon 2011; $42(6): 807-13$

2. Durso FT, Sethumadhavan A. Situation Awareness: Understanding 6 Dynamic Environments. Hum Factors 2008; $50(3)$ : 442-48.

3. Endsley MR. Toward a theory of situation awareness in dynamic Systems. Hum Factors 1995; $37(1): 32-64$.

4. Henderson JM. Human gaze control during real-world scene 10 perception. Trends Cogn Sci 2003; 7 (11): 498-504.

5. Howell DC. Statistical methods for psychology, Belmont: Wadsworth; 12 2013: $346-52$.

6. Iqbal ST, Adamczyk PD, Zheng XS, Bailey BP. Towards an index of 14 opportunity: Understanding changes in mental workload during task execution. In: Proceedings of the SIGCHI Conference on Human Factors in Computing Systems; NY: ACM; 2005: $311-20$.

7. Johnson A, Proctor RW. Attention: theory and practice, London: Sage Publications, Inc.; 2004: 254-84.

8. Jones DG, Endsley MR. Sources of situation awareness error in aviation. Aviat Space Environ Med 1996; 67: 507-12. 
$457-66$.

2 10. Kowler E. Attention and eye movements. In Krauzlis R. Ed.

4

5

6

8 Encyclopedia of Neuroscience. Elsevier Ltd: Amsterdam; $2008: 605-16$

11. Kowler E. Eye movements: The past 25 years. Vision Res 2011; 51: $1457-83$.

12. Lavine RA, Sibert L, Gokturk M, Dickens B. Eye-tracking measures and human performance in a vigilance task. Aviat space Environ Med 2002; 73: 367-72.

13. Li WC, Yu CS, Li LW, Greaves M. Pilots' eye movement patterns during performing air-to-air mission. In: Proceedings of the 31st European Association for Aviation Psychology Conference; Valletta, Malta; 2014: 265-73.

14. Lipshitz R, Klein G, Orasanu J, Salas E. Taking stock of naturalistic decision making. J Behav Decis Mak 2001; 14(5): $331-52$.

15. Merwe K, Dijk H, Zon R. Eye movements as an indicator of situation awareness in a flight simulator experiment. Int J Aviat Psychol 2012; $22(1)$ : 78-95.

16. Morelli F, Burton PA. The impact of induced stress upon selective attention in multiple object tracking. Mil Psychol 2009; $21(1): 81-97$.

17. National Transportation Safety Board. Descent below Visual 
Glidepath and Impact with Seawall, Asiana Airlines Flight 214 (No. AAR1401). Washington DC: NTSB; 2013.

18. Orasanu J. Crew collaboration in space: a naturalistic decision making perspective. Aviat Space Environ Med 2005; 76(6, Suppl. ) :B154-63.

19. Partala T, Surakka V. Pupil size variation as an indication of affective processing. Int J Hum Comput Stud 2003; 59(1-2): $185-98$.

20. Rognin L, Grimaud I, Hoffman E, Zeghal K. Assessing the impact of a new instruction on air traffic controller monitoring tasks. In: Proceedings of the International Conference on Human-Computer Interaction in Aeronautics; Toulouse, France; 2004: 197-203.

21. Salvucci DD, Goldberg JH. Identifying fixations and saccades in eye-tracking protocols. Duchowski AT. ed. In: Proceedings of the 2000 Symposium on Eye Tracking Research \& Applications; Florida: ACM; 2000: 71-8.

22. Sarter NB, Mumaw RJ, Wickens CD. Pilots' monitoring strategies and performance on automated flight decks: An empirical study combining behavioral and eye-tracking data. Hum Factors 2007; $48(3): 347-57$.

23. Wang JT. Pupil dilation and eye tracking. In Mecklenbeck MS, Kuehberger A, Ranyard R. Eds. A Handbook of Process Tracing Methods for Decision Research: A Critical Review and User's 
Guide. NY: Psychology Press; 2010: 185-204.

2 24. Zhao M, Gersch TM, Schnitzer BS, Dosher BA, Kowler E. Eye 3 movements and attention: The role of pre-saccadic shifts of attention in perception, memory and the control of saccades. Vision Res 2012; 74: 40-60. 
TABLE I

\begin{tabular}{|c|c|c|}
\hline Variables & Groups & Frequencies \\
\hline \multirow{4}{*}{ Age } & $25-30$ & $13(35.1 \%)$ \\
\hline & $31-35$ & $11(29.7 \%)$ \\
\hline & $36-40$ & $7(18.9 \%)$ \\
\hline & $41-45$ & $6(16.2 \%)$ \\
\hline \multirow{5}{*}{ Rank } & Lieutenant & $1 \quad(2.7 \%)$ \\
\hline & Captain & $16(43.2 \%)$ \\
\hline & Major & $9(24.3 \%)$ \\
\hline & Lieutenant Colonel & $10 \quad(27 \%)$ \\
\hline & Colonel Above & $1(2.7 \%)$ \\
\hline \multirow{5}{*}{ Qualification } & Combat ready & $13(35.1 \%)$ \\
\hline & Two fighter team leader & $4(10.8 \%)$ \\
\hline & Four fighter team leader & $9(24.3 \%)$ \\
\hline & Daytime back seat instructor & $2(5.4 \%)$ \\
\hline & Training instructor & $9(24.3 \%)$ \\
\hline \multirow{5}{*}{ Total Flight Hours } & 500 and less & $3(8.1 \%)$ \\
\hline & $501-1000$ & $13(35.1 \%)$ \\
\hline & $1001-1500$ & $11(29.7 \%)$ \\
\hline & $1501-2000$ & $4 \quad(10.8 \%)$ \\
\hline & 2001 and above & $6(16.2 \%)$ \\
\hline
\end{tabular}

2

TABLE I. SUBJECTS' DEMOGRAPHIC VARIABLES . 
TABLE II

\begin{tabular}{|c|c|c|c|c|c|c|c|c|c|}
\hline \multirow{2}{*}{ Variables } & \multirow{2}{*}{ Tasks } & \multirow{2}{*}{$\mathrm{M}$} & \multirow{2}{*}{$\mathrm{SD}$} & \multirow{2}{*}{$\mathrm{N}$} & \multicolumn{5}{|c|}{ T-Test } \\
\hline & & & & & t & $d f$ & $p$ & $S E$ & Cohen's d \\
\hline \multirow{2}{*}{ Fixations } & $\mathrm{AA}$ & 8.0 & 2.2 & \multirow{2}{*}{37} & \multirow{2}{*}{-2.521} & \multirow{2}{*}{36} & \multirow{2}{*}{.016} & \multirow{2}{*}{0.44} & \multirow{2}{*}{-0.624} \\
\hline & AS & 9.2 & 1.6 & & & & & & \\
\hline \multirow{2}{*}{$\begin{array}{c}\text { Fixation } \\
\text { duration } \\
\text { (msec) }\end{array}$} & $A A$ & 751 & 543 & \multirow{2}{*}{37} & \multirow{2}{*}{3.263} & \multirow{2}{*}{36} & \multirow{2}{*}{.002} & \multirow{2}{*}{89.67} & \multirow{2}{*}{0.748} \\
\hline & AS & 458 & 111 & & & & & & \\
\hline \multirow{2}{*}{$\begin{array}{l}\text { Pupil size } \\
\quad\left(\text { pixel }{ }^{2}\right)\end{array}$} & $\mathrm{AA}$ & 23990 & 7703 & \multirow{2}{*}{37} & \multirow{2}{*}{-1.922} & \multirow{2}{*}{36} & \multirow{2}{*}{.063} & \multirow{2}{*}{913.33} & \multirow{2}{*}{-0.252} \\
\hline & AS & 25746 & 6173 & & & & & & \\
\hline \multirow{2}{*}{$\begin{array}{c}\text { Saccade } \\
\text { duration } \\
\text { (msec) }\end{array}$} & $\mathrm{AA}$ & 196 & 215 & \multirow{2}{*}{37} & \multirow{2}{*}{-2.297} & \multirow{2}{*}{36} & \multirow{2}{*}{.028} & \multirow{2}{*}{30.82} & \multirow{2}{*}{-0.372} \\
\hline & AS & 267 & 163 & & & & & & \\
\hline \multirow{2}{*}{$\begin{array}{c}\text { Saccade } \\
\text { velocity } \\
\text { (pixels/sec) }\end{array}$} & $A A$ & 948 & 319 & \multirow{2}{*}{37} & \multirow{2}{*}{-1.308} & \multirow{2}{*}{36} & 199 & 4560 & $-0 \quad 214$ \\
\hline & AS & 1007 & 224 & & & & & & \\
\hline
\end{tabular}




\begin{tabular}{|c|c|c|c|c|c|c|c|c|}
\hline Variables & Tasks & Phases & $M$ & $S D$ & $d f$ & $F$ & $p$ & $\eta 2 p$ \\
\hline \multirow{6}{*}{$\begin{array}{c}\text { Percentage of } \\
\text { fixations } \\
\text { (arcsine values) }\end{array}$} & \multirow{3}{*}{$\mathrm{AA}$} & S & 37.57 & 5.72 & \multirow{3}{*}{36} & \multirow{3}{*}{5.75} & \multirow{3}{*}{.005} & \multirow{3}{*}{.138} \\
\hline & & A & 35.11 & 2.96 & & & & \\
\hline & & $\mathrm{L}$ & 32.94 & 5.37 & & & & \\
\hline & \multirow{3}{*}{ AS } & $\mathrm{S}$ & 33.54 & 4.68 & \multirow{3}{*}{36} & \multirow{3}{*}{6.29} & \multirow{3}{*}{.003} & \multirow{3}{*}{.149} \\
\hline & & A & 37.62 & 3.93 & & & & \\
\hline & & L & 34.23 & 4.35 & & & & \\
\hline \multirow{6}{*}{$\begin{array}{l}\text { Fixation } \\
\text { duration } \\
\quad(\text { msec })\end{array}$} & \multirow{3}{*}{$\mathrm{AA}$} & S & 612 & 487 & \multirow{3}{*}{36} & \multirow{3}{*}{5.39} & \multirow{3}{*}{.007} & \multirow{3}{*}{.130} \\
\hline & & A & 702 & 515 & & & & \\
\hline & & $\mathrm{L}$ & 938 & 881 & & & & \\
\hline & \multirow{3}{*}{ AS } & S & 332 & 71 & \multirow{3}{*}{36} & \multirow{3}{*}{18.48} & \multirow{3}{*}{.000} & \multirow{3}{*}{.339} \\
\hline & & A & 462 & 145 & & & & \\
\hline & & $\mathrm{L}$ & 580 & 270 & & & & \\
\hline \multirow{6}{*}{$\begin{array}{l}\text { Pupil size } \\
\quad\left(p i x e l^{2}\right)\end{array}$} & \multirow{3}{*}{$\mathrm{AA}$} & S & 21960 & 10132 & \multirow{3}{*}{36} & \multirow{3}{*}{7.57} & \multirow{3}{*}{.001} & \multirow{3}{*}{.174} \\
\hline & & A & 23864 & 8762 & & & & \\
\hline & & $\mathrm{L}$ & 26147 & 6449 & & & & \\
\hline & \multirow{3}{*}{ AS } & S & 23997 & 6180 & & & & \\
\hline & & A & 27105 & 6565 & 36 & 38.82 & .000 & .519 \\
\hline & & $\mathrm{L}$ & 26136 & 6152 & & & & \\
\hline & & S & 239 & 332 & & & & \\
\hline & $\mathrm{AA}$ & A & 167 & 188 & 36 & 1.34 & .269 & .036 \\
\hline Saccade duration & & L & 183 & 270 & & & & \\
\hline$(\mathrm{msec})$ & & S & 457 & 288 & & & & \\
\hline & AS & A & 204 & 198 & 36 & 29.06 & .000 & .447 \\
\hline & & L & 141 & 170 & & & & \\
\hline & & S & 970 & 438 & & & & \\
\hline & $\mathrm{AA}$ & A & 983 & 438 & 36 & 0.68 & .510 & .019 \\
\hline Saccade velocity & & $\mathrm{L}$ & 891 & 437 & & & & \\
\hline (pixels/sec) & & S & 829 & 368 & & & & \\
\hline & AS & A & 1045 & 328 & 36 & 7.87 & .001 & .179 \\
\hline & & L & 1148 & 394 & & & & \\
\hline
\end{tabular}

TABLE III. ANOVA Of EYE MOVEMENTS at THREE OPERATIONAL PHASES: SEARCHING (S), AIMING (A) and LOCK-ON (L) during the TASKS of AIR-to-AIR (AA) and AIR-to-SURFACE (AS). 


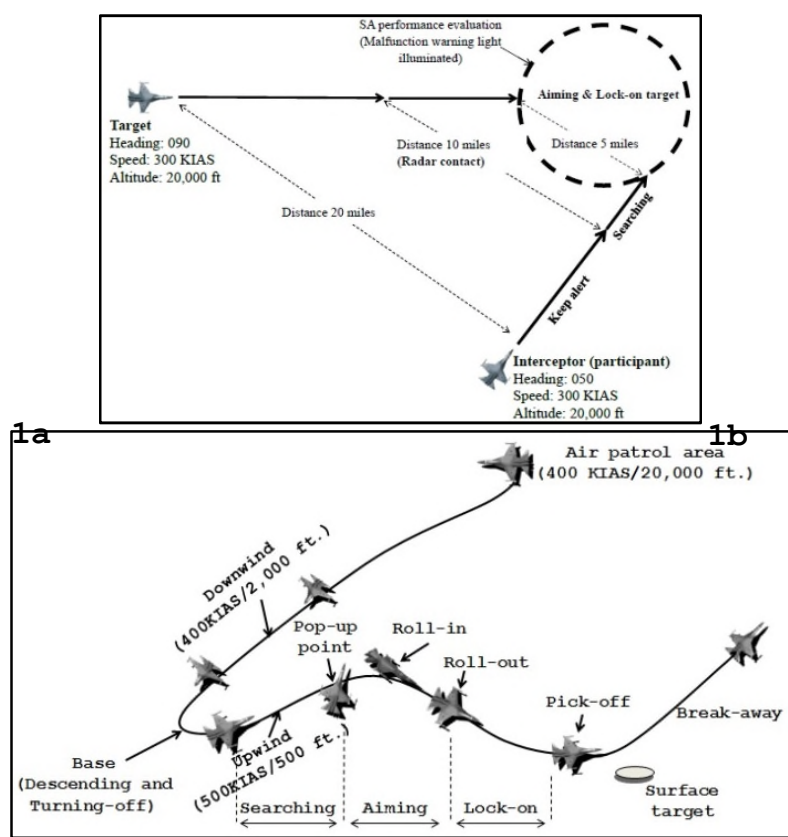

3
4

FIGURE 2

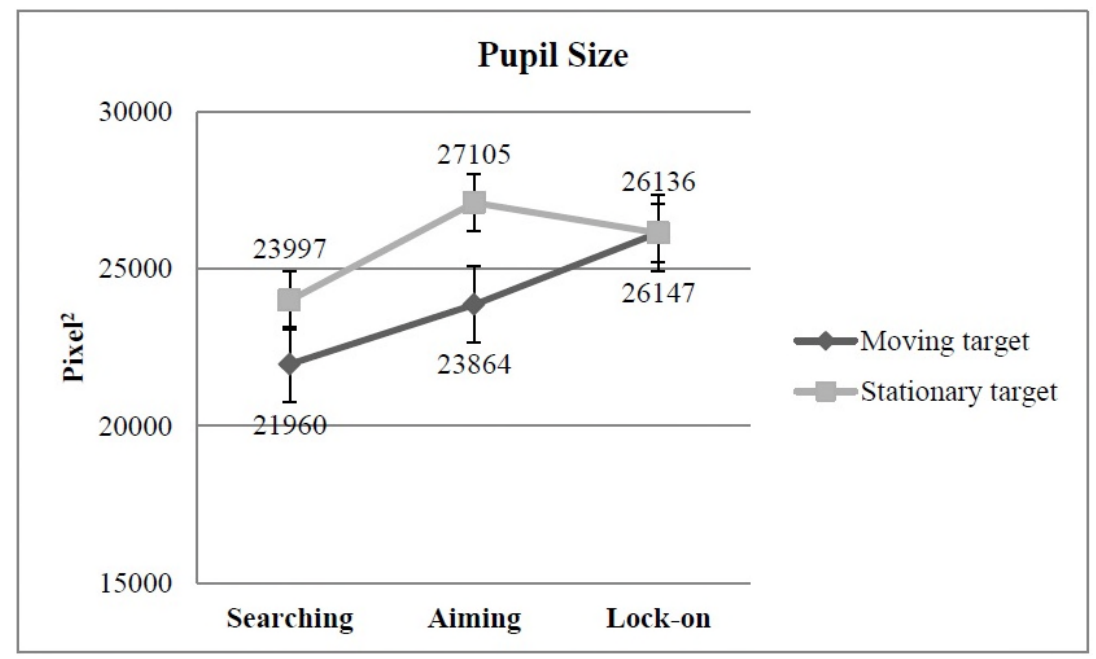

11 FIGURE 2. PILOTS' PUPIL DILATION among THREE OPERATIONAL PHASES WHILE 13 PURSUING a MOVING TARGET and STATIONARY TARGET. THE BIGGEST PUPIL 14 DILATION IS DURING the AIMING PHASE WHEN PURSUING a STATIONARY TARGET 15 INDICATED the HIGHEST WORKLOAD. 
FIGURE 3. PILOTS' SACCADE DURATION at THREE OPERATIONAL PHASES WHILE PURSUING the MOVING TARGET and the STATIONARY TARGET. SEARCHING PHASE SHOWS the LONGEST SACCADE DURATION for BOTH TASKS, and PURSUING STATIONARY TARGET DEMOSTRATED SIGNIFICANT LONGER SACCADE DURATION THAN MOVING TARGET at SEARCHING PHASE.

FIGURE 4

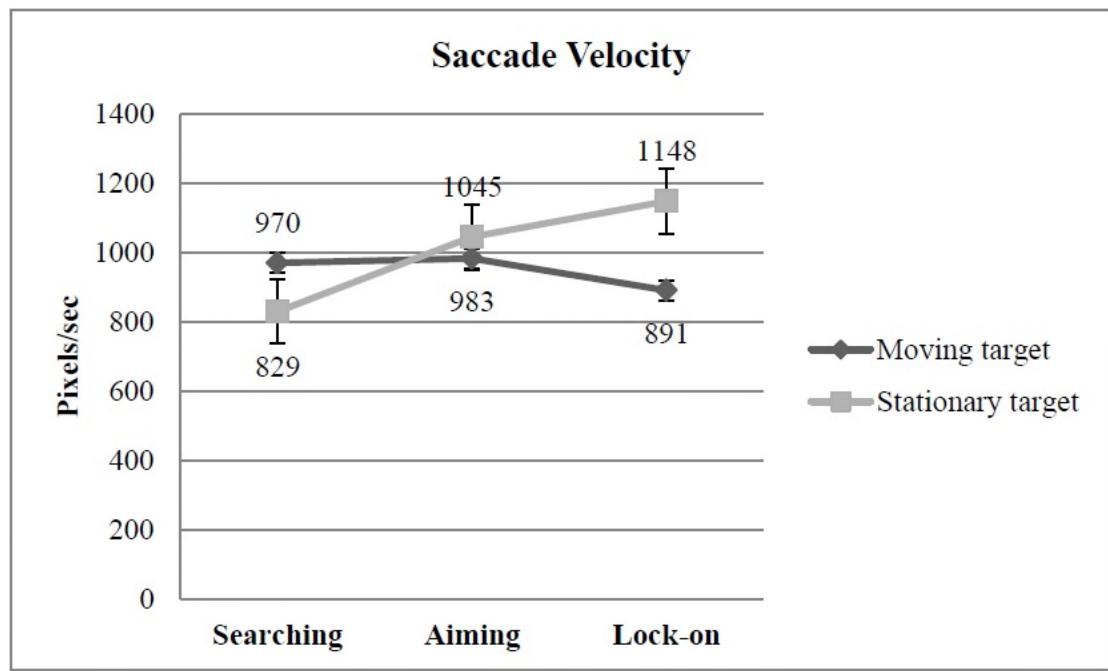

FIGURE 4. PILOTS' SACCADE VELOCITY at THREE OPERATIONAL PHASES WHILE PURSUING the MOVING TARGET and the STATIONARY TARGET. THE FASTEST SACCADE VELOCITY OCCURS at the LOCK-ON PHASE On PURSUING a STATIONARY TARGET. IT IS SIGNIFICANTLY FASTER THAN PURSUING a MOVING TARGET. 
2016

pÿPilot s attention distributions between chasing a moving target and a stationary target

\author{
Li, Wen-Chin
}

Aerospace Medical Association

pÿWen-Chin Li, Chung-San Yu, Graham Braithwaite and Matthew Greaves. Pilot s attention distributions between chasing a moving target and a stationary target. Aerospace Medicine and Human Performance, Volume 87, Number 12, December 2016, pp989-995

https://dspace.lib.cranfield.ac.uk/handle/1826/10704

Downloaded from Cranfield Library Services E-Repository 